livraisons

d'Histoire

de l'Architecture

\section{Livraisons de l'histoire de l'architecture}

$17 \mid 2009$

Émotions patrimoniales I

\title{
Les églises en France sous le Second Empire : deux illustrations du type idéalisé
}

The church architecture constructed during the Second Empire in France: two illustrations of idealized architectural type

Kirchenbau in Frankreich im Second Empire : zwei Beispiele des damals idealisierten Typs

\section{Sanghoon Kang}

\section{OpenEdition}

Journals

Édition électronique

URL : http://journals.openedition.org/lha/214

DOI : $10.4000 /$ lha. 214

ISSN : 1960-5994

Éditeur

Association Livraisons d'histoire de l'architecture - LHA

Édition imprimée

Date de publication : 10 juin 2009

Pagination : 107-117

ISSN : 1627-4970

Référence électronique

Sanghoon Kang, "Les églises en France sous le Second Empire : deux illustrations du type idéalisé », Livraisons de l'histoire de l'architecture [En ligne], 17 | 2009, mis en ligne le 10 juin 2011, consulté le 10 décembre 2020. URL : http://journals.openedition.org/lha/214 ; DOI : https://doi.org/10.4000//ha.214

Ce document a été généré automatiquement le 10 décembre 2020.

Tous droits réservés à l'Association LHA 


\section{Les églises en France sous le Second Empire : deux illustrations du type idéalisé}

The church architecture constructed during the Second Empire in France: two

illustrations of idealized architectural type

Kirchenbau in Frankreich im Second Empire : zwei Beispiele des damals

idealisierten Typs

Sanghoon Kang

1 L'affirmation que l'Académie des Beaux-Arts énonce au milieu du XIX ${ }^{\mathrm{e}}$ siècle sur le rapport étroit que l'art moderne entretient avec les modèles de la Grèce et de Rome trouve sa pertinence dans le cadre du fameux débat animé par Raoul-Rochette à propos du style gothique ${ }^{1}$. Elle permet au concept d'éclectisme de prendre de l'ampleur. C'est, en effet, sous le Second Empire que les partisans de l'éclectisme apportent, en France, notamment à Paris, de véritables réponses architecturales aux défis lancés par les archéologues. Bien qu'elles se signalent par leur force d'expression, on leur a reproché d'être souvent «figées dans la gangue du pastiche et de la copie, simples et médiocres recommencements ${ }^{2}$ ", tout en s'interdisant de les reconnaître comme un courant stylistique de l'époque. Il faut reposer la question : en quoi les réponses des éclectiques se démarquent-elles ? Peuvent-elles au moins exprimer une nouveauté aussi pertinente que celle que Raoul-Rochette reconnaît aux architectures de la Renaissance ? De quelle originalité témoignent-elles, comme architectures religieuses, au sein de l'architecture publique, dans une époque où la notion d'originalité permet de rendre compte du bouleversement social et artistique, de se reconnaître et de se revendiquer comme un tournant déterminant, voire une césure, dans l'évolution de l'art et de l'architecture?

2 Ces questions prennent tout leur sens lorsqu'on observe sous l'angle de leur diversité stylistique les églises bâties sous le Second Empire : comment évaluer d'une manière rationnelle la valeur de ces édifices et des architectes éclectiques qui les ont conçues? 


\section{«Cathédrale » et « basilique »}

3 L'intensification du mouvement de construction d'églises en France depuis la Monarchie de Juillet témoigne de la nécessaire adaptation du catholicisme aux nécessités de l'époque : la restauration sociale et religieuse, l'étude du passé idéalisé en rapport avec la redécouverte du Moyen Age et des origines du christiannisme, les mutations démographiques ainsi que la question du prix de revient. Les églises sous le Second Empire révèlent cette nécessité d'adaptation, ainsi que l'ambivalence du souci d'économie et de la liberté de style passant par le pastiche et de nouvelles tentatives en matière de construction, comme les structures métalliques. À Paris, elles sont édifiées par les plus grands architectes de l'époque et se montrent souvent exubérantes, alors que, dans les départements, elles subissent plus directement des contraintes économiques et s'en tiennent souvent aux " plans types »3 . En étudiant l'église de SaintAugustin à Paris, par exemple, peut-être l'une des réponses les plus représentatives du Second Empire aux besoins liturgiques et urbains ainsi qu'aux intentions sociales, on comprend l'image que doit produire l'église française de l'époque. L'étude des diverses solutions que le Second Empire a apportées permet de comprendre ce qui caractérise l'architecture publique de l'époque, quand on la met en rapport avec la naissance de l'esthétique comme discipline philosophique, laquelle signale une mutation radicale de la représentation du beau au XIX ${ }^{\mathrm{e}}$ siècle.

4 Les églises françaises font d'abord référence à deux types idéalisés : «cathédrale » et «basilique ». Le XIX ${ }^{\mathrm{e}}$ siècle n'a pas seulement inventé le concept de « cathédrale » à la française $e^{4}$, mais aussi celui de "basilique $»^{5}$. L'analyse de la signification politique, sociale et cultuelle de la « cathédrale » au XIX ${ }^{\mathrm{e}}$ siècle conduit à constater que le contenu de cette notion est marqué par une restructuration religieuse qui découle de nouveaux besoins pratiques et liturgiques et par un pur intérêt stylistique. L'affirmation de l'utilité conduit à revisiter ces deux types, " cathédrale » et "basilique », dans le projet de construire une base de réflexion qui permettrait de réévaluer la valeur des églises de l'époque en évitant des jugements sommaires. Les édifices qui s'inspirent de l'un et l'autre de ces types manifestent clairement le projet de rétablir une architecture religieuse qui, du moins artistiquement, s'oriente vers une forme d'originalité individuelle.

5 L'évolution de type "cathédrale» se manifeste d'abord par l'invention de la « cathédrale idéale » néo-gothique viollet-le-ducienne et surtout par un autre type qui résulte de la combinaison de toutes les références. La complexité du plan des cathédrales reconstruites, restaurées et nouvellement conçues, qui en résulte semble constituer une synthèse stylistique originale. La question majeure que l'on peut se poser au sujet du nouveau visage du type « cathédrale » concerne cette transformation fonctionnelle, qui apparait comme un nouveau motif stylistique, s'inscrit dans le grand courant qui fait alors la part belle à l'éclectisme.

6 La cathédrale de la Major à Marseille de Léon Vaudoyer, l'une des grandes réalisations architecturales du règne de Napoléon III, concrétise parfaitement ce visage nouveau du type « cathédrale ». Elle est porteuse d'une conception laïque et bourgeoise qui ne place certainement pas la religion au premier rang de la vie intellectuelle dans la cité mais la considère comme une facette parmi d'autres d'égale valeur. D'autres constructions font appel au néo-roman et au néo-romano-byzantin, même si l'on ne peut guère en citer d'exemples majeurs. Ces édifices expriment, nous semble-t-il, la volonté de créer un 
type idéalisé de « cathédrale » conçu d'après le type « basilique » qui s'oppose à celui de cathédrale néo-gothique archéologique. Il est donc essentiel de savoir comment ces exemples du type «cathédrale » se rapportent étroitement à l'évolution du type «basilique ». Le type «basilique » résulte du mouvement de redécouverte des origines chrétiennes et sert précisément de base, voire de prototype, à plusieurs cathédrales nouvellement construites et à diverses églises paroissiales de la seconde moitié du XIX siècle.

\section{« Cathédrale idéale » conçue d'après le type « basilique »}

7 La « cathédrale idéale » de Vaudoyer ${ }^{6}$, jugée « historiciste », ne peut pas être considérée comme un cas parfait sous l'angle esthétique car la question de l'adéquation du type à la fonction religieuse reste un point controversé dans la conception de Vaudoyer. Contrairement au type «idéal» viollet-le-ducien dont le principe s'affranchit des circonstances du lieu et de l'environnement naturel et social, la cathédrale de la Major à Marseille (ill. 1) ne met pas entre parenthèse la question du lieu et de la fonction.

\section{III.1 : Cathédrale de la Major, Marseille, par Léon Vaudoyer, 1852-1874}

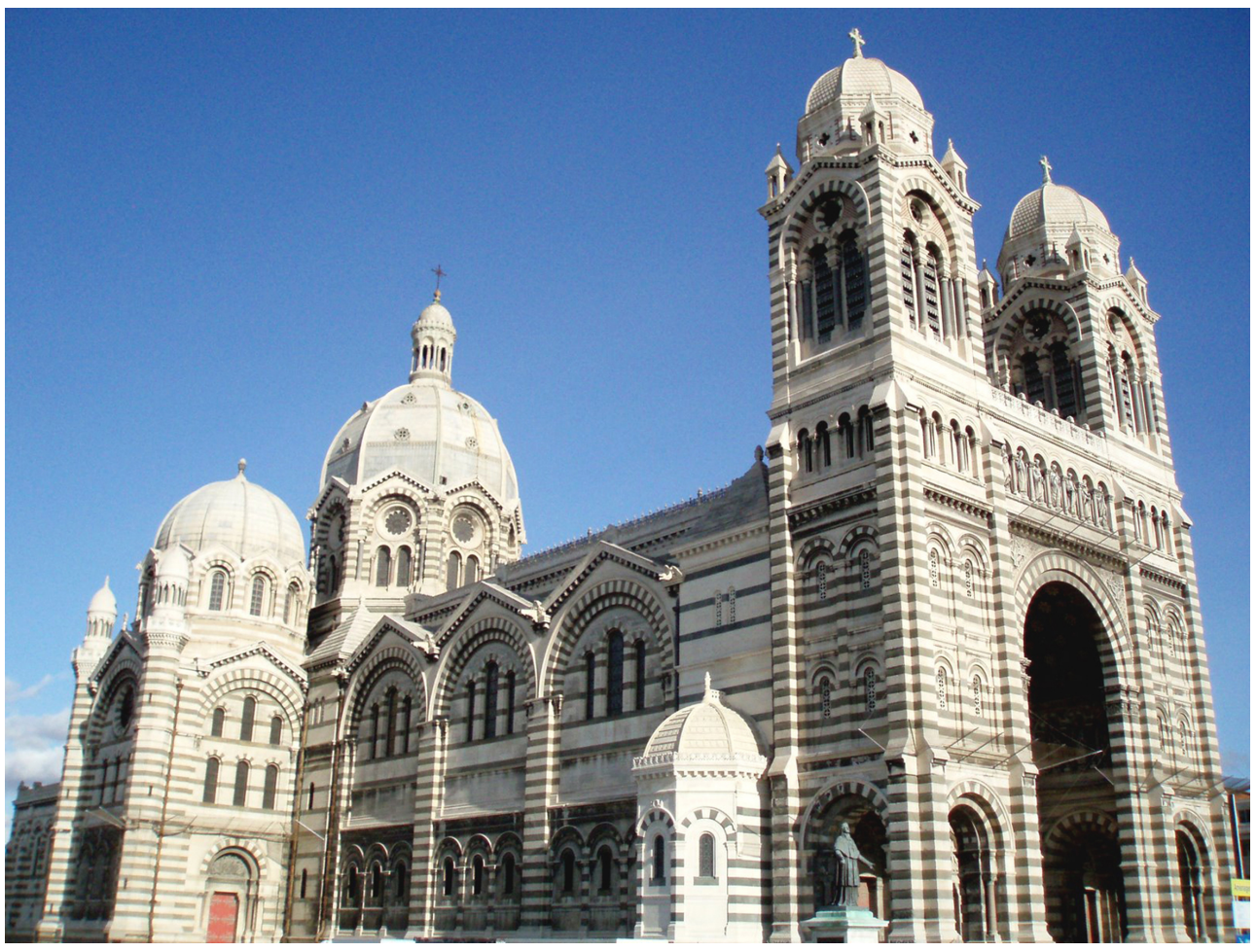

Cl. Sanghoon Kang.

Vaudoyer se préoccupe davantage d'articuler les composantes de la masse extérieure où se juxtaposent des éléments empruntés à différents styles historiques bien plus que d'instaurer un dialogue cohérent avec la fonction de l'intérieur ou de répondre à l'exigence de provoquer une émotion religieuse. Le dessin de la façade avec son grand arc et la bichromie des volumes extérieurs, par exemple, fournissent de purs effets plastiques qui s'imposent à l'environnement urbain. On voit par cet exemple combien 
l'intérêt religieux est subordonné à la conception artistique qui résulte, en l'occurrence, d'une réponse synthétique aux exigences de la « la trilogie labroustienne ${ }^{7}$ ", du lieu, des usages et du climat. À Marseille, la relation entre l'intérieur, avec le sentiment religieux qui en découle, et l'extérieur, avec son ambition plastique, ne parait pas aux yeux de l'architecte une question essentielle : il s'agit dans l'esprit de Vaudoyer d'inventer un type d'édifice qui établisse un rapport nouveau entre la tradition et l'originalité de façon à construire une synthèse de l'histoire de l'architecture chrétienne. Pourtant, les formes et les significations de ce nouveau courant du type « cathédrale » demeurent aussi peu compréhensibles par le public que ne le fut la controverse provoquée par le type "basilique» de l'église de SainteGeneviève de Soufflot. Au total, la cathédrale de la Major marque l'époque comme fruit d'un historicisme idéalisé et signale une évolution épistémologique dans le projet d'unir l'architecture, le lieu et la religion chrétienne sans rompre pour autant avec l'héritage du passé.

Dans le contexte des créations de l'École diocésaine, le dessein qu'exprime Vaudoyer pour résoudre « le fameux problème du type catholique ${ }^{8}$ » se distingue par la référence qu'il donne du type basilical: une vaste salle, de forme allongée, des colonnades à l'intérieur, un dôme et une croisée plus importante pour le transept se combinent dans une grande église byzantine où l'on trouve l'écho de la basilique antique et paléochrétienne. Dans l'Encyclopédie nouvelle, il est affirmé que la «basilique», avec son principe d'arcades retombant sur les colonnes, servit de base à l'architecture de tous les styles et de tous les temps 9 . Le «type catholique » s'en serait particulièrement inspiré à la fin de l'Antiquité ${ }^{10}$.

La combinaison des arcades avec les colonnes a été très apprécié par Quatremère de Quincy et, à sa suite, par Aubin-Louis Millin en $1806^{11}$. Pour eux, le type "basilique » s'inscrit dans la pure traduction du beau absolu par le moyen de l'imitation ${ }^{12}$. À la différence de Quatremère de Quincy, Vaudoyer, en revanche, s'appuie sur le changement historique et la nécessité de s'adapter à des contextes nouveaux, ainsi qu'en témoigne le processus de création de la cathédrale de la Major à Marseille. Cet édifice ne participe pas de la création d'un nouveau type de cathédrale, mais résulte de l'application rigoureuse des déductions méthodiques et originales qui s'attachent à la définition d'un concept d'église française à partir du type basilical historique.

\section{« Basilique » adaptée au type « cathédrale idéale »}

11 Viollet-le-Duc, qui estime sage et heureux le projet définitif de Vaudoyer ${ }^{13}$, définit le terme «basilique » dans le Dictionnaire raisonné et réaffirme la conclusion de Vaudoyer en illustrant la nature des métamorphoses historiques du type "basilique ». Il ajoute que la disposition générale des églises modernes auxquelles le Pape attribue le titre «basilique " ne rappelle en rien celle de la basilique primitive. Séparable donc de l'esprit de la basilique ancienne au point de vue architectonique, la basilique moderne se traduit comme "l'appropriation d'un édifice antique à un besoin moderne, non comme la réalisation d'un programme arrêté $»^{14}$.

Les interprétations de la notion de "basilique " sont diverses, de la basilique primitive à la conception "idéale» de Boullée: à travers la recherche d'un style propre à l'époque, c'est le développement théorique d'un type "basilique» à partir des problématiques invoquées par les rendus des élèves de l'École des beaux-arts depuis le 
début du siècle que reflètent bien les églises sous le Second Empire. La première occasion d'y réfléchir se présente lors du concours d'émulation en $1801^{15}$ : tout en respectant le plan traditionnel, les élèves expriment leur volonté marquée de réadaptation archéologique dont la question du couvrement de la croisée donne un cas symbolique. L'organisation générale de nombreuses cathédrales en France correspond à la manière dont les élèves conçoivent le type "cathédrale idéale » dans le rendu du grand prix de Rome de l'époque. La cathédrale projetée à Ajaccio, par exemple, se marque clairement par son respect archéologique, et la question de couverture y vient au premier plan.

La cathédrale d'Ajaccio, fondée sur la recherche de références historiques, présente un clocher Renaissance et un dôme surhaussé. Empruntant des éléments très classiques, le projet semble traduire une notion vague d'inspiration éclectique. Il diffère peu du courant des projets des élèves, lesquels, en plaçant un grand dôme à la croisée, insoucieux du lieu, de l'usage, des matériaux et d'autres considérations architecturales, tombent souvent dans l'abstrait stylistique et typologique en passant du type "basilique " au type "cathédrale idéale ", comme le font le projet d'Uchard en 1838 intitulé " église cathédrale » (ill. 2 et 3 ) et celui de Thomas, élève d'Hippolyte Lebas, l'auteur de Notre-Dame de Lorette, réalisé en 1845 sous le titre « église cathédrale pour une ville» (ill. 4).

III. 2 : Façade principale du projet en 1838 pour une « église cathédrale », par Toussaint-FrançoisJoseph Uchard

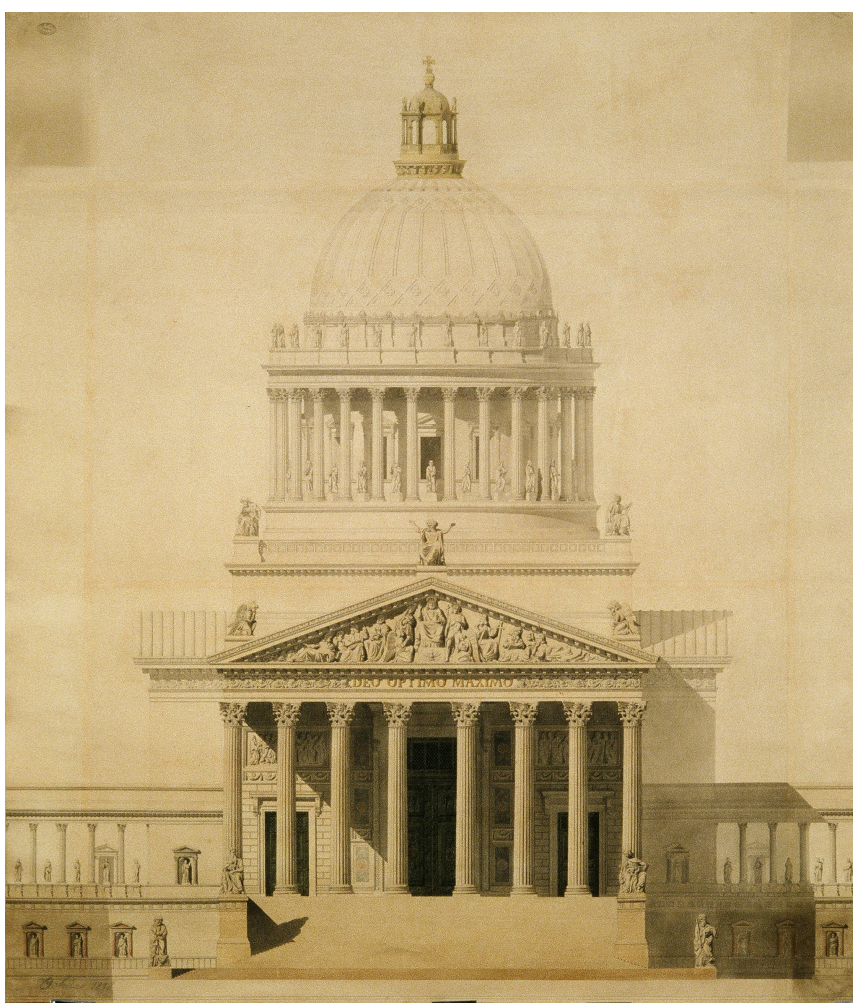

École nationale supérieure des beaux-arts

Cl. Sanghoon Kang. 
III. 3 : Coupe longitudinale du projet en 1838 pour une « église cathédrale », par Toussaint-FrançoisJoseph Uchard

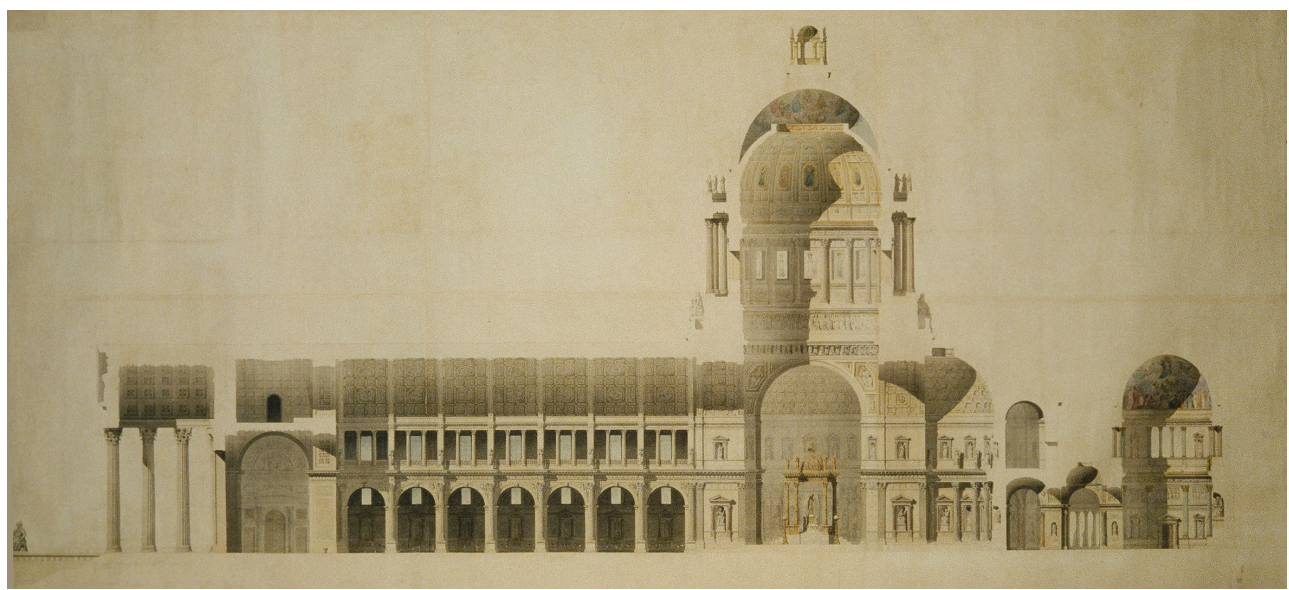

École nationale supérieure des beaux-arts

Cl. Sanghoon Kang

III. 4 : Façade principale du projet en 1845 pour une " église cathédrale pour une ville capitale ", par Félix Thomas

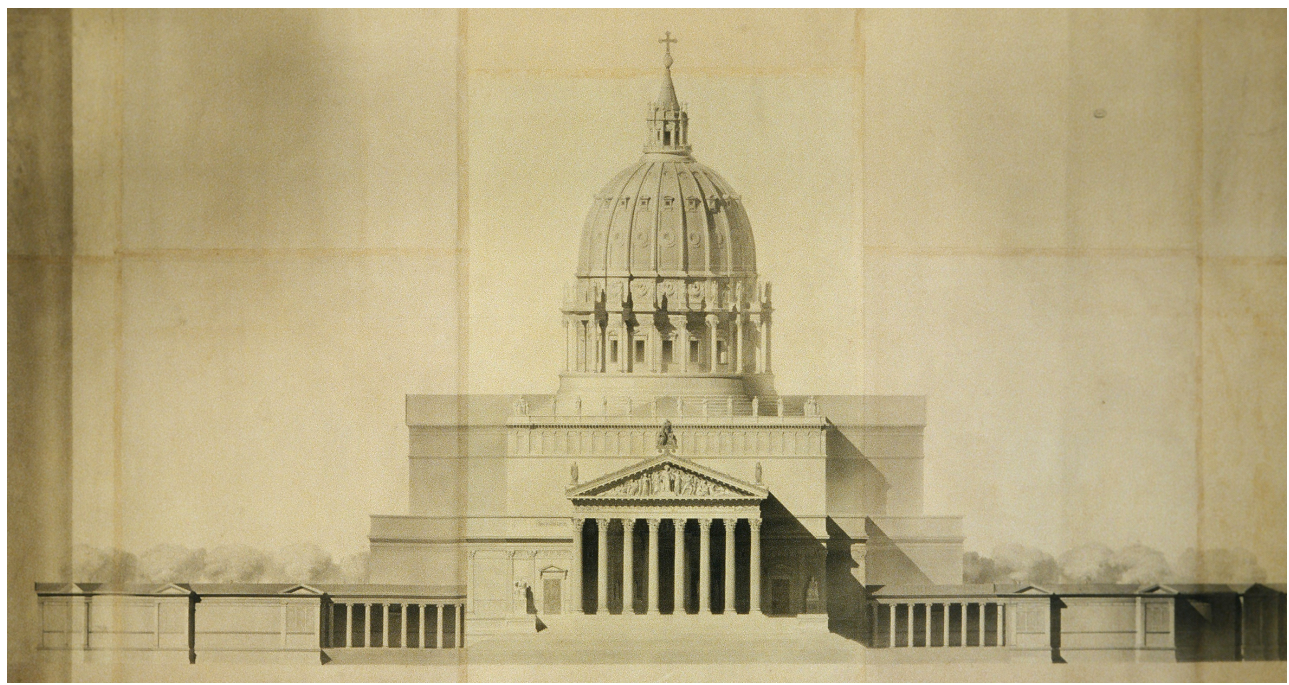

École nationale supérieure des beaux-arts

Cl. Sanghoon Kang.

On y trouve une disposition analogue à celle de la basilique Sainte-Geneviève, qui permet de constater que l'œuvre de Soufflot donne sans doute le point de départ de la réflexion de l'auteur de la nouvelle "cathédrale » et que la tradition du rêve néoclassique à forte charge symbolique poursuit ses variations sur le projet de "basilique " ou de métropole de Boullée. Les rendus des élèves invoquent certainement la question de l'appropriation d'un édifice à un besoin moderne et prennent en compte les nouveaux besoins liturgiques et les exigences nouvelles : l'économie et le grand motif urbain, indispensable à la ville du Second Empire. Dans ce cadre, l'évolution du type «basilique » s'exprime d'une manière à la fois implicite et explicite dans des édifices tels que Saint-Augustin (ill. 5) à Paris, l'un des fleurons de l'architecture religieuse du Second Empire. 


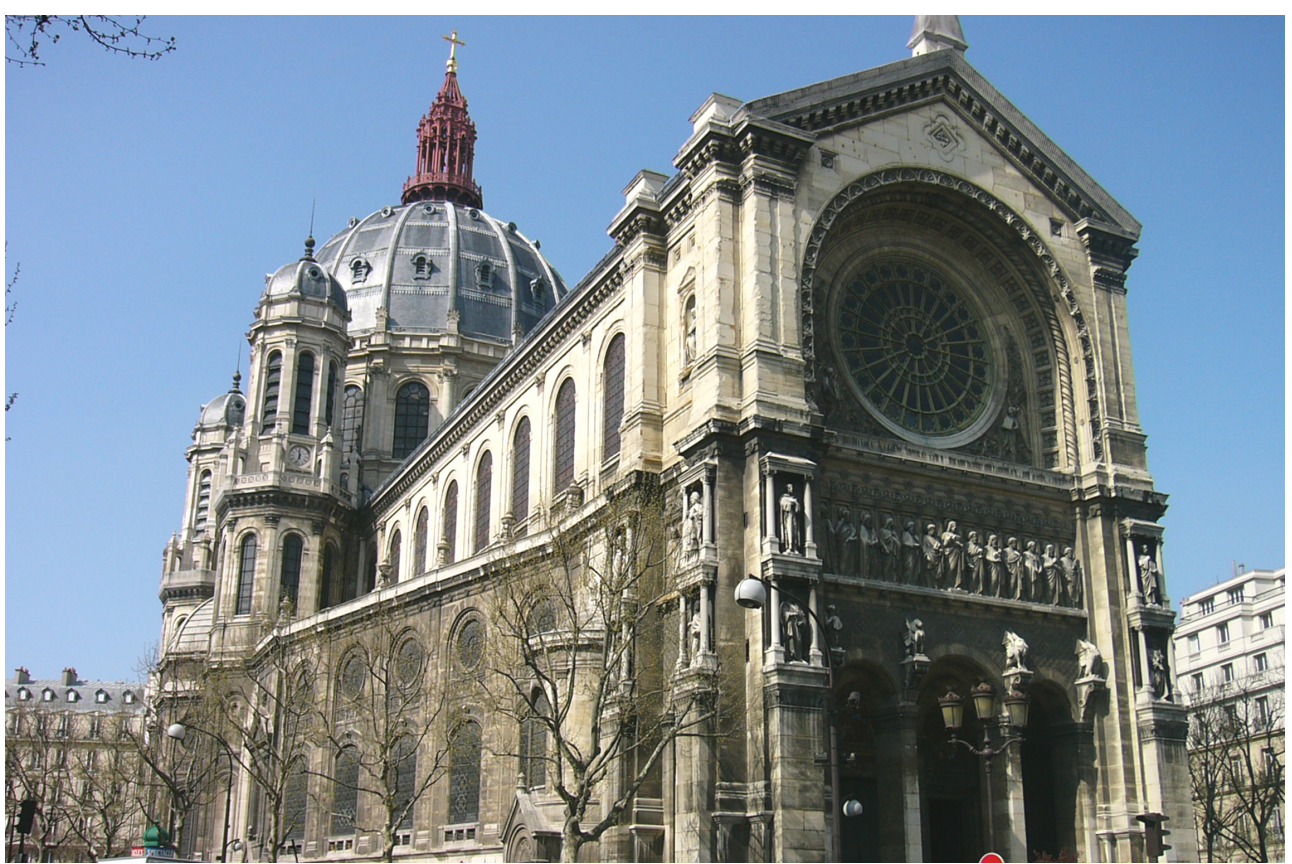

CL. SANGHOON KANg

En utilisant le fer et la fonte dans la construction de cet édifice, non seulement par souci d'économie, mais aussi pour répondre à des exigences structurelles et esthétiques, Victor Baltard entreprend de prouver que le nouveau matériau se prête au caractère d'un édifice du culte. Très sincèrement religieux et ami de prêtres éminents, il réalise ici son rêve : élever une église "à la gloire de Dieu ${ }^{16}$. Il croit en trouver les éléments nécessaires dans le fer et la fonte. A la fois, donc, pour remplir les fonctions d'une véritable structure porteuse et pour la faire participer à la décoration, Baltard utilise la fonte dont il possède l'expérience depuis la construction des Halles centrales. Cependant, ce n'est pas seulement que son intérêt pour les structures métalliques, mais la forme du terrain en trapèze, qui conduit au tracé d'une seule et non de trois nefs et le force à trouver le moyen de faire une voûte sans contreforts, ce que, seul, le grand arc en fer permettait de réaliser. Au total, l'expression franche et fonctionnelle du bâtiment confirme la nouveauté des conceptions de l'architecte, notamment son refus de dissimuler le fer derrière des placages trompeurs de maçonnerie ainsi qu'y incite alors le préjugé qui persiste en matière d'architecture religieuse à l'encontre du métal.

L'articulation des volumes intérieurs témoigne, en particulier, de la volonté de l'architecte de créer et de faire œuvre de liberté en faveur de la convenance religieuse. Sans doute pour conférer tout l'honneur au centre eucharistique, Baltard élève la plateforme du chœur à un niveau beaucoup plus élevé que la nef de façon que le maîtreautel apparaisse clairement depuis toutes les parties de l'église et dispose au-dessus la coupole. À ces espaces majeurs clairement hiérarchisés malgré l'absence d'espace de transition entre la nef et le chœur, s'ajoutent des chapelles en forme de parallélépipède dont la dimension s'élargit progressivement à mesure qu'on s'approche du sanctuaire. On en éprouve forcément la fausse perspective, mais ceci semble donner plus d'importance au sanctuaire bien éclairé, partie de l'édifice sur laquelle Baltard veut, en effet, concentrer l'effet visuel et symbolique. L'idée de l'architecte de fermer les 
chapelles par des murs biais très minces, qui résultent principalement de la disposition structurelle, permet d'ailleurs de disposer des vitraux de dimensions et de formes variées, y compris celle du cercle. Le niveau de la lumière naturelle, par conséquent, varie et crée un effet inédit.

Moins d'audace, mais de "l'imagination active $»^{17}$ selon le terme de Viollet-le-Duc : la disposition générale de l'intérieur est considérée donc comme un passage obligé qu'exige la circonstance de l'emplacement. Elle imprime ainsi des caractères différents par la variation inédite du plan, le jeu de lumière et l'expression franche de la structure en fer avec ses demi-colonnes de fonte ornées et polychromes plaquées sur les piles de pierre. Il en découle un paysage de l'élévation intérieure, l'une des voies qu'envisageait l'architecture religieuse moderne à la suite de Soufflot, Boullée, Durand et Vaudoyer. La grande nef, vaste espace intérieur de caractère inédit, et la coupole signalent un édifice monumental,. Pour autant, le style de Saint-Augustin n'est pas adapté aux fonctions religieuses. Le choix du style incarne la résolution de l'architecte qui voulait à la fois concevoir une œuvre artistique adaptée à l'îlot parisien isolé des édifices environnants, et implanter dans la capitale française un édifice grandiose qui constituât une synthèse des références historiques et des courants artistiques. Contrairement à l'église de SaintPierre de Montrouge, l'autre église emblématique du Second Empire qui correspond au développement d'un quartier très peuplé, Saint-Augustin se montre ambitieux et transparent dans le choix du symbole d'autant qu'elle se situe dans le centre de Paris et près de monuments majeurs. Baltard voulait-il produire l'image idéale d'une église digne de la «basilique » comme celle qui s'attache à la basilique Sainte-Geneviève de Soufflot? Il est certain que Baltard tient à ce que son église soit un prototype de l'époque, une » basilique » moderne adaptée au type "cathédrale idéale », aux besoins modernes, aux usages des grands rassemblements religieux et à l'îlot parisien, bref, une autre version de la trilogie des fonctions « labroustiennes».

Le défaut de ce courant architectural tient au fait que l'architecte réduit l'effet de l'ensemble de l'édifice en donnant à chaque partie un caractère distinct. L'articulation des corps de l'extérieur de Saint-Augustin rappelle plus ou moins l'apparence du formalisme classique, qui n'est pourtant attachée qu'à l'effet plastique, en énonçant un rapport faible avec l'intérieur. D'ailleurs, l'on peut supposer que Baltard, soucieux de la pureté archéologique, cherche à exprimer une apparence qui correspond mieux à des formes « basiliques » adaptées au type " cathédrale idéale », et s'inspire des images des architectures classiques.

Le dôme à lanterne, digne de ceux de Sainte-Marie-des-Fleurs à Florence et de SaintPierre de Rome, indique clairement sa destination en tant que symbole urbain et crée une partie intégrante de la silhouette artistique du centre de Paris, mais la convenance propre du dôme à la destination de Saint-Augustin se concrétise-t-elle dans une forme déterminée ? Viollet-le-Duc énonce une critique qui résume cette interrogation :

En définitive, nous croyons que les convenances propres à la destination de l'édifice ont été pour peu de chose dans le choix du parti architectural adopté. La question dominante paraît avoir été de produire, par la construction d'une immense coupole, un effet monumental déterminé, sauf à s'accommoder des dispositions qui allaient résulter de ce parti pour l'installation des services appelés à trouver place dans l'édifice. C'est ainsi, nous croyons, que ces services ne s'accusent à l'extérieur par aucune disposition spéciale, conforme à la nature de leurs exigences, et qu'il a fallu, pour les assurer tant bien que mal, diviser les vides dans lesquels on les a logés, par des planches et des cloisons qui dénaturent la construction monumentale. Sous ce 
rapport, la construction de l'église de Saint-Augustin ne s'écarte pas des errements qui ont prévalu jusqu'à ce jour dans l'architecture moderne ${ }^{18}$. d'équilibre entre l'emploi des nouveaux matériaux et la volonté de donner au monument un aspect propre à susciter un sentiment religieux, en renouvelant la manière dont Boullée avait conçu son dôme, avec son parti pris esthétique d'élévation et les «tableaux» majestueux de l'espace dont il avait pourvu l'intérieur de sa «basilique ${ }^{19}$. Baltard essaie d'induire du nouveau à partir des références historiques, mais, en fait de création, son manque d'audace dans l'emploi du fer et un jeu plastique trop soumis à la contrainte de l'emplacement paralysent l'invention de formes nouvelles. Au total, il n'arrive pas à déconstruire ou altérer les modèles des «basiliques » historiques, en adapter les éléments à la nouvelle situation et au bout du compte, en transcender le caractère. Ce courant architectural se forme dans un contexte similaire à celui de la "cathédrale idéale» de Vaudoyer, qui reste malheureusement, comme le caractérise Jean-Michel Leniaud, un hapax de l'époque.

\section{NOTES}

1. Raoul-Rochette et Eugène Viollet-le-Duc : «Considérations sur la question de savoir s'il est convenable, au XIX ${ }^{\mathrm{e}}$ siècle, de bâtir des églises en style gothique » dans Annales archéologiques, $\mathrm{t}$. IV, 1846, pp. 325-353 et Jean-Michel LENIAUD, « Une tentative de police esthétique : l'Académie des beaux-arts et les "Considérations sur la question de savoir s'il est convenable, au XIX siècle, de bâtir des églises en style gothique" 1846 ", La Révolution des signes. L'art à l'église (1830-1930), Paris, Cerf, 2007, 429 p., p. 57-84. Voir aussi Chantal Bouchon et al., Ces Églises du XIX ${ }^{e}$ siècle, Amiens, Encrage, 1993, 270 p.

2. Bruno FOUCART, «Comment peut-on aimer une église du XIX siècle ou de la réhabilitation du pastiche » dans Monuments historiques, $\mathrm{n}^{\circ}$. 3, 1974, p. 64-65.

3. Anatole de BAUDOT, Églises des bourgs et villages, Paris, 1867.

4. Jean-Michel LENIAUD, Les Cathédrales au XIXe siècle, Paris, Economica, 1993, 988 p., p. 23 et p. 15-24.

5. Du même, « Les basiliques de pélerinage en France », La Révolution des signes, op.cit., p. 179-190.

6. Notamment l'étude de Barry BERGDOLL, Léon Vaudoyer ; Historicism in the age of industry, New York, The MIT Press, 1994, 416 p. et David VAN ZANTEN, Designing Paris, New York, The MIT Press, 1987, 338 p., p. 137-175.

7. Jean-Michel LENIAUD, Les Cathédrales au XIX siècle, op. cit., p.508.

8. Ibid, p.494, 507 ; Barry Bergdoll, Léon Vaudoyer, Historicism in the age of industry, p. 232. Arch. nat., 246 AP 14, lettre du 14 mai 1845.

9. Pierre Leroux, Jean Reynaud, « Basilique », dans Encyclopédie nouvelle, vol. 2, Paris, 1836, p. 470 : «Sans juger jusqu'à quel point le système d'arcade sur les colonnes est admissible comme bonne construction ou comme forme architecturale, nous ferons remarquer que ce type inventé par les chrétiens est celui qui servit de base à l'architecture byzantine et arabe; puis, par suite, à l'architecture romane et à celle dite gothique, et qu'après avoir été adopté par les maîtres de la renaissance, il est parvenu jusqu'à nous.

10. « Architecture », ibid, p. 775. 
11. Aubin-Louis Millin, «Basilique », dans Dictionnaire des beaux-arts, vol. 1, 1806, p. 114-117.

12. Antoine Quatremère de Quincy, «Basilique », dans Encyclopédie méthodique, Architecture, 3 vol., Paris, 1788-1825, t. I, p. 223-231.

13. Arch. nat., $\mathrm{F}^{19} 7740$, Comité des inspecteurs généraux des édifices diocésains, séance du 5 mai 1855, rapport fait au comité par Viollet-le-Duc: «M. Vaudoyer en envoyant sa nouvelle étude jointe au projet approuvé [...] a été amené à donner à la nef plus de largeur, aux alentours de la coupole centrale et du sanctuaire des dispositions plus simples et en rapport avec les pesanteurs et poussées, à ses élévations et coupes, un caractère d'unité. Ce système d'architecture admis, et il convient parfaitement au climat et aux matériaux de Marseille, on ne pouvait en faire une application plus sage et plus heureuse. »

14. Eugène Viollet-le-Duc : «Basilique », dans Dictionnaire raisonné de l'architecture, t. II, 1863, p. 165.

15. Arch. nat., $\mathrm{AJ}^{*} / 52 / 95$, concours d'émulation de première classe, rendu, 1801 : « Basilique ou Temple chrétien ", première médaille ; Hippolyte Lebas, deuxième médaille; Hector Lefuel Gase.

16. Sur la notice biographique de Victor Baltard et l'église de Saint-Augustin, voir notamment Louis Arnould, "Victor Baltard, 1805-1874», dans Bulletin des amis de Sceaux, année 12-13, 1936-1937 ; Henri Delaborde, "Architecte contemporain, Victor Baltard ", dans Revue des deux mondes, t. 2, 15 avril, 1874 ; Paul Sédille, «Victor Baltard, architecte », dans Gazette des beaux-arts, t. 9, mai, 1874 ; Louis Arnould indique que le goût des choses religieuses pousse Baltard dans la restauration d'un grand nombre d'églises parisiennes. Baltard rêvait d'élever un temple à la gloire de Dieu, fondé sur son expérience pratique des nouveaux matériaux acquise dans la réalisation des Halles et sur ses sentiments religieux : «Un jour, lors de l'achèvement d'un de ces pavillons, la dernière poutrelle de fer mise en place, l'architecte fut invité par les ouvriers, suivant leur habitude, à arroser le bouquet placé par eux tout en haut: il fait monter des bouteilles de vin, il gravit encore une fois les échelles de l'échafaudage et là, le verre en main, entre ciel et terre, il lève le bras en disant seulement : " Mes amis, à la gloire de Dieu ! ", simples mots hardis, qui peignent parfaitement et sa cordialité pour tous et l'élévation naturellement religieuse de ses sentiments. »

17. Eugène Viollet-le-Duc, Entretiens sur l'architecture, sixième entretien, Paris, A. Morel, 1873, p. 175-180.

18. Eugène-Louis Viollet-le-Duc, "Église de Saint-Augustin », dans Gazette des architectes et du bâtiment, t. VI, $\mathrm{n}^{\circ} 1,1868$, p. 6.

19. Étienne-Louis Boullée, «Basilique », dans Architecture, essai sur l'art, textes réunis et présentés par Jean-Marie Pérouse de Montclos, Hermann, Paris, 1968, 187 p., p. 79-97.

\section{RÉSUMÉS}

Moment d'un débat intensif sur le style en architecture, le Second Empire apparaît comme l'époque où le concept d'éclectisme prend de l'ampleur. En France, et notamment à Paris, ses partisans apportent alors de véritables réponses architecturales mais on s'interdit de reconnaitre ces réalisations comme appartenant à un courant stylistique majeur, alors même qu'elles se signalent par leur force d'expression.

En quoi les réponses des éclectiques se démarquent-elles ? De quelle originalité témoignent-elles à une époque où la notion d'originalité permet de rendre compte du bouleversement social et artistique et donne à un mouvement les moyens de se reconnaître et de se revendiquer comme 
un tournant déterminant, voire comme une véritable césure, dans l'évolution de l'art et de l'architecture?

Les églises bâties sous le Second Empire en France s'intègrent dans ce débat et ces questions prennent tout leur sens lorsqu'on les observe sous l'angle de leur diversité stylistique. Une recherche sur le rapport entre les deux types idéalisés de "cathédrale " à la française et de «basilique », auxquels les églises de l'époque font référence, constitue la base de cet article. L'invention, à l'époque, de ces deux concepts, aux évolutions étroitement liées, témoigne en faveur de la pertinence à les placer au centre d'une réflexion sur l'évaluation rationnelle de la valeur architecturale de ces bâtiments religieux.

The Second Empire is a period when eclectic style in architecture reached in its blossom. The enthusiasm on the eclectic style in architecture in France, particularly in Paris makes it possible to produce architectural solutions full of the creative imagination. There is, however, a general tendency in discussions on architectural style not to regard eclecticism as the representative style that denotes the $19^{\text {th }}$ century.

Then, in what original aspects can be understood the eclectic style in architecture in the period when the establishment of the notion of originality, as important criteria of social aspect, of artistic practice and of aesthetic appreciation, signifies a radical change in the evolution of art and architecture?

These questions will be answered upon a close observation on the characteristics of French churches constructed during the Second Empire, which are closely related to this debate on architectural style. The article investigates the method to evaluate the characteristics of the church architecture in a rational way; and pays attention to the two idealized types of religious architecture, "cathedral" and "basilica", to which the church architecture of the $19^{\text {th }}$ century has reference. It is essential to realize that the $19^{\text {th }}$ century invented the concept of "cathedral" type conceived in a French manner and that of "basilica", and also to notice that the evolution of the one type is often closely implicated with the other. This will attest the feasibility of bring out these two architectural types for understanding the value of the church architecture of the Second Empire.

Das Second Empire wurde das Zeitalter einer intensiven Debatte über Stil und Architektur. So verstärkte sich in dieser Zeit der Begriff «Eklektizismus ». Die Anhänger dieses Stils bauten in Frankreich, hauptsächlich in Paris, bemerkenswerte architektonische Beispiele. An diesen Bauten wurden jedoch die Merkmale eines eigenen Stiles gar nicht anerkannt, obwohl besonders starke Ausdrucksformen vor Augen geführt wurden. Die unter dem Second Empire gebauten Kirchen sind repräsentativ für diese Debatte, besonders wenn man sie in ihrer stilistischen Diversität ansieht.

Worin unterscheidet sich denn die Antwort der Eklektiker? Worin liegt deren Originalität in einer Zeit, in der die Vorstellung von Originalität mit der sozialen und ästhetischen Umwälzung eng verbunden war? Inwiefern kann diese Bewegung als eine besondere Wende, sogar eine wahrhafte Zäsur in der Entwicklung der Kunst und der Architektur bezeichnet werden?

Der Aufsatz unterscheidet in den religiösen Kirchenbauten dieser Zeit zwei idealisiserte Typen, einerseits den Dom à la française, andererseits die Basilika.Diese zwei schon in der damaligen Zeit angenommenen Bezeichnungen, die sich eng aneinander entwickelten, erweisen sich heute als besonders zweckmäßige Kriterien, wenn man die rationale Bewertung dieser religiösen Architektur einzuschätzen versucht . 
INDEX

Index chronologique : XIXe siècle, époque contemporaine

Mots-clés : église

Keywords : church

Schlüsselwörter : Kirche

\section{AUTEUR}

\section{SANGHOON KANG}

Sanghoon Kang, né en 1963, a obtenu une maîtrise d'architecture à l'université de New York et sa licence d'ingénieur mécanique à l'université de Boston. Il a soutenu un DEA et une thèse de doctorat à l'université de Paris I-Panthéon-Sorbonne en 2001 (sujet : L'originalité de l'architecture religieuse au XIX ${ }^{e}$ siècle en France). Il travaille actuellement dans un cabinet d'architectes à Séoul et donne un cours d'histoire de l'architecture dans plusieurs universités en Corée du sud.

Adresse électronique : sono6375@naver.com 